\title{
An Investigation into Long-acting Reversible Contraception: Use, Awareness, and Associated Factors
}

\author{
Mojgan Zendehdel ${ }^{1}$, Shayesteh Jahanfar ${ }^{2 *}$, Zainab Hamzehgardeshi ${ }^{3,4}$, Ensiyeh Fooladi ${ }^{5}$
}

\author{
${ }^{1}$ Reproductive and Sexual Health Research Centre, School of Nursing and Midwifery, Mazandaran University of Medical Sciences, Sari, Mazandaran, IRAN \\ ${ }^{2}$ School of Health Sciences, Central Michigan University, Health Professions Building 2242, Mount Pleasant, MI, 48859, USA \\ ${ }^{3}$ Sexual and Reproductive Health Research Center, Mazandaran University of Medical Sciences, Sari, IRAN \\ ${ }^{4}$ Associate Professor, Department of Reproductive Health and Midwifery, Nasibeh Faculty of Nursing and Midwifery, Mazandaran University of Medical Sciences, Sari, \\ IRAN \\ ${ }^{5}$ Women's Health Research Program, School of Public Health and Preventive Medicine, Monash University, Melbourne, AUSTRALIA \\ *Corresponding Author: jahan2s@cmich.edu
}

Citation: Zendehdel, M., Jahanfar, S., Hamzehgardeshi, Z., \& Fooladi, E. (2020). An Investigation into Long-acting Reversible Contraception: Use, Awareness, and Associated Factors. European Journal of Environment and Public Health, 4(2), em0039. https://doi.org/10.29333/ejeph/7837

\section{ARTICLE INFO}

Received: 3 Feb. 2020

Revised: 14 Feb. 2020

Accepted: 15 Feb. 2020

\begin{abstract}
Objectives: We aimed to investigate the prevalence, awareness, perceived reliability, and factors associated with the use of long-acting reversible contraception among reproductive-aged, married women and men in Iran.

Methods: In this cross-sectional study, 1520 men and women between 15-49 years of age who attended public health centers in Tehran were surveyed.

Results: About $85 \%$ of the respondents reported that they were already familiar with intrauterine contraception and $61.9 \%$ with Medroxyprogesterone Acetate. The majority of women respondents had not considered IUDs (57.2\%) or DMPA (59.1\%) as reliable methods. As for men, IUDs $(53.8 \%)$ or DMPA (39.8\%) were considered as reliable methods of contraception. Moreover, a higher number of women thought it was better to refrain from using IUDs (60.3\%) or DMPA (61.5\%) than men [IUDs (53.4\%) or DMPA (40.2\%)]. Those who in the younger age group (18-40 years old), had younger partners (18-40 years old), had education beyond a high school diploma and had no history of unwanted pregnancy (58\%) were less likely to use LARC.

Conclusions: Men and women are familiar with LARC (Long-acting reversible contraception) methods, but few believe that these methods are reliable. Demonstration of the performance of long-term methods by health personnel will increase the belief and trust of women and men in longer-lasting ways of contraception.
\end{abstract}

Keywords: long-acting reversible, contraception, use, awareness, associated factors

\section{INTRODUCTION}

Contraception is widely used in Iran, with a prevalence rate of $81.5 \%$ (Motlaq et al., 2013; Simbar, 2010). The most common types of contraception include modern methods (oral contraceptive methods, long-acting methods, condoms, tubectomy, and vasectomy), used by $59.2 \%$ of contraception users, and traditional methods such as withdrawal and safe interval, used by $22.3 \%$ of users (Moosazadeh et al., 2014; Motlaq et al., 2013).

Unwanted pregnancy is a global public health issue that leads to serious health, economic, and social consequences for women and their families (Brown \& Eisenberg, 1995), both worldwide and across the large and diverse Asia-Pacific region. This region is of particular interest since the rate of unwanted pregnancy is high (about 44\%) (Sedgh et al., 2014).
The rate of unwanted pregnancy in Iran varies to a great extent, from 10.5\% in Semnan to 49.3\% in Rasht 2016 (Motlaq et al., 2013; Namazi, 2015; Zaheri et al., 2015).

Long-acting reversible contraception (LARC) methods include etonogestrel contraceptive implants, copper intrauterine devices (IUDs) and levonorgestrel-releasing intrauterine systems (DMPA). Over the past four decades, modern LARC methods have been available in Iran (Jalal Abbasi-Shavazi \& McDonald, 2006; Mehryar et al., 2001; Simbar, 2012). However, only $10.6 \%$ of women aged 15-49 use an IUD and only 2.8\% use DMPA (Motlaq et al., 2013; Simbar, 2010). According to the World Health Organization's (WHO) medical eligibility criteria for contraceptive use (Curtis, 2010), LARC does not show obvious contraindications. Furthermore, almost all women are eligible for using implants and intrauterine contraception (Group, 2009). LARC also has some advantages over other contraceptive methods, some of which include high effectiveness and worriless coitus. Therefore, 
users will not require motivation and adherence are guaranteed at every sexual encounter. Moreover, LARC is reversible and does not require frequent visits for resupply (Committee, 2009). Compared with other contraceptive methods, LARC has higher rates of continuation and satisfaction (Likis, 2014; Peipert et al., 2011). According to reports, LARC was not widely used in Iran in 2012 (IUD =10.5\%, DMPA=2.5\%) when compared with other high-income countries such as the UK and the USA. It is estimated that about $8 \%$ of women of reproductive age in the UK and $10 \%$ in the USA use LARC (Eeckhaut et al., 2014). The increased use of LARC can reduce unwanted pregnancy and induced abortion (Committee, 2009; Holton et al., 2016).

The total fertility rate (TFR) in Iran dropped from 6.5 in 1960 to 1.6 in 2012, which is well below the targeted value of 2.2. Therefore, the WHO presented the World's Leading Birth Control Country Award to Iran in 2001. However, this award led to unexpected ramifications because families became reluctant to have children and people showed a high willingness to get married at older ages. These reasons factor into why the TFR decreased to a great extent, lower than intended by earlier policymakers. There is an urgent need to pay attention to this figure, as the TFR will fall below the replacement level if it continues to drop. This brought new challenges, some of which include an increased dependency ratio, and problematic economic and social concerns. Additionally, the policy change led to other concerns, such as limitations on the distribution of contraceptive methods; a lack of education in health clinics about what contraceptive methods should or should not be used, and on how to use permitted contraceptive methods; and the removal of vasectomy and tubectomy methods from the list of permissible contraceptive methods (Erfani, 2013). Given the lack of training on contraception methods in Iranian public health centers, as well as the overuse or underuse of contraception and its impact on society, it is important to research in this field to raise awareness and identify the perceived reliability of long-term methods in men and women of reproductive age.

This research thus aims to assess the prevalence, awareness, perceived reliability, and factors associated with the use of LARC among reproductive-aged, married women and men aged 15-49.

\section{MATERIALS AND METHODS}

\section{Study Design}

The current study has a cross-sectional design. The Ethics Committee approved this study of Mazandaran and Tehran Medical Sciences (IR.MAZUM.REC.96.3019).

\section{Sample and Recruitment}

The recruited sample consisted of married women and men of reproductive age (15-49 years old), living in the southern part of Tehran. Subjects were referred to the health centers to receive health care services. All the participants signed a written consent form. The exclusion criteria included being a bachelor, widowed, divorced, or unable to respond to the questions of the survey due to a lack of proper verbal communication in the Persian language.

\section{Sampling Method}

A stratified random sampling method was utilized by Mojgan Zendehdel, who randomly selected 62 out of 110 health centers in the southern part of Tehran. Participants referring to these centers were selected by using convenient sampling.

\section{Calculating the Sample Size}

According to the census received from the Planning and Management Organization of Tehran, men and women aged $15-49$ constituted a population of 898,577 people in 2016 . The sample size was calculated based on a recent study, according to which the prevalence rate of unwanted pregnancies was 30\% (Motlaq et al., 2013). Overall, 1013 women and 507 men participated in this study. In addition, the questionnaire was completed for all of the participants, and no samples were excluded.

$$
n=\frac{p(1-p) Z \frac{\alpha}{2}}{d^{2}}
$$

Where

$$
\begin{gathered}
p=30 \% \\
d=5 \% \\
Z \frac{\alpha}{2}=1.96
\end{gathered}
$$

Substituting (2) into (1) results in:

$$
n=\frac{3.84 * 0.3 * 0.7}{(0.05)^{2}}=336
$$

$$
\begin{aligned}
& 336(18-25) \text { women } \\
& 336(26-40) \text { women } \\
& 336(41-49) \text { women } \\
& 336^{*} 3=1008 \text { sample women } \\
& 336 * 3=1008 \text { sample men }
\end{aligned}
$$

However, the number of married men aged (15 - 25) was very low because of the increased marriage age. Moreover, the numbers of men were referred to health centers were substantially smaller than women, which resulted in the smaller men samples. As a matter of fact, we considered 507 men and 1013 women samples in our study (in overall $=507+1013=1520$ ).

\section{Data Collection}

The inquiry began in January 2017 and ended in October 2017. The researcher randomly selected married women and men who were 15-49 years old. They were visiting the health centers in order to receive services, such as family planning, pregnancy care, monitoring of children's health, middle-age care (from 30 to 59 years old), dentistry, occupational health services, nutrition, etc. Having completed the informed written consent, the questionnaire was completed by the participants in a completely private environment. The time required to complete each questionnaire was 25 to 35 minutes.

\section{Research Tools}

The questionnaire consisted of several sections as follows: 
10 questions were related to demographic information, such as age of the participant and her/his spouse, sex, the education level of the participant and her/his spouse $(0=$ diploma and less, 1 = diploma and beyond $)$, employment $(0=$ no, $1=$ yes $)$, number of times married $(0=$ once, $1=$ more than once), satisfaction with health status $(0=$ poor, $1=$ good $)$, financial status $(0=$ bad $1=$ good, $)$, religion $(0=$ Shia Islam, $1=$ Sunni Islam), and participants' perception of the importance of religion in fertility choices $(0=$ not important, $1=$ important).

One question was included for fertility knowledge $(0=$ poor, 1 = good). 6 questions were related to the awareness of the LARC methods: "Are you familiar with IUDs?", "Are IUDs reliable?”, "Have/would you consider(ed) using IUDs?”, “Are you familiar with DMPA?", "Is DMPA reliable?" and" Have/would you consider(ed) using DMPA?”

Use of other preventive methods was addressed using six questions: use of current contraception $(0=$ no, $1=$ yes), having consulted with health professionals for contraception over the past two years $(0=$ no, $1=$ yes $)$, talking comfortably with health care providers about contraception $(0=$ not comfortable, $1=$ comfortable), thinking about the participant's life right now, how important is it to you/your spouse to avoid pregnancy $(0=$ not important, $1=$ very important), and the convenience of talking to the spouse regarding the use and selection of the contraceptive method $(0=$ uncomfortable, $1=$ comfortable).

The record of pregnancy history was also gathered using three questions: a history of pregnancy $(0=$ no, $1=y e s)$, history of unwanted pregnancy $(0=$ none, $1=$ at least once $)$ and history of abortion $(0=$ never, $1=$ at least once) .

\section{Long-acting Reversible Contraception}

Two types of LARC methods are available in Iran: intrauterine contraception (both copper and hormonal) and contraceptive injections. The contraceptive implant is not available in Iran. The present study evaluated the participants' awareness of, attitudes towards, and use of intrauterine contraception and contraceptive injections.

The two types of LARC were addressed with three main questions: "Have you ever heard anything about this method?", "Do you think this method is reliable?", and "Would you consider using this method? The participants were supposed to answer the questions by the following options: 'Yes', 'No', or 'I don't know'. In order to analyze the first question (awareness of LARC), the options were classified as either 'Yes' or 'No and I don't know'. Few participants answered, 'I don't know'. In order to analyze the perceived reliability, all three options were included. The same questions were posed both for women and men.

\section{Data Management and Analysis}

All participants who completed the questionnaires were included in the analysis. Quantitative data were analyzed using IBM SPSS Statistics 22. Descriptive statistics were used to summarize the data.

The researchers aimed to investigate the associations between the characteristics of respondents and the perceived reliability and use of LARC. Accordingly, $\mathrm{X}^{2}$ tests were conducted in order to investigate the associations between the characteristics of respondents and the perceived reliability and use of both types of LARC (intrauterine and DMPA).

The outcome variables were divided into three categories (Yes, No, I don't know). These characteristics were investigated by multinomial logistic regression analyses, which estimated odds ratios for the relationship between individual characteristics and the use and perceived reliability of LARC. We aimed to determine significant differences between the use of LARC and perceived reliability, using the characteristics of respondents. The option 'Yes' was regarded as the reference category.

For each sex (women and men), four multinomial logistic regression analyses were conducted: (1) perceived reliability of IUDs (dependent variable) using the characteristics of respondents (independent variables); (2) perceived reliability of DMPA (dependent variable) using the characteristics of respondents (independent variables); (3) the use of IUDs (dependent variable) using the characteristics of respondents (independent variables); and (4) the use of DMPA (dependent variable) using the characteristics of respondents (independent variables).

\section{RESULTS}

\section{Sample and Response}

Of the 3857 patients referred to the health centers, 1520 married men and women were enrolled in the study and gave their responses using a questionnaire during face-to-face interviews.

\section{Awareness and Use of LARC}

Most of the participants were familiar with IUDs (84.9\%) and DMPA (61.9 \%). However, more than half of the participants (56.1\%) reported that the IUD or DMPA methods (52.6\%) were not reliable and that they would not consider using them (58\% and $54.4 \%$, respectively). Women were much more familiar with IUDs (92.1\%) compared to men $(70.6 \%, p=$ 0.001) and DMPA (Women: 74\%, men: 37.7\%; $p=0.001$ ). Furthermore, women were more likely than men to regard IUDs (women 34.5\%, men 20.7\%; $\mathrm{x}^{2}(n=1520) 29.9, p=0.001$ ) or DMPA (women 15.4\%, men 5.7\%; $\mathrm{x}^{2}(n=1520) 12.2, p=$ 0.001 ) as reliable. Women were also more likely than men to consider using an intrauterine method (women 32.8\%, men $\left.20.7 \% ; \mathrm{x}^{2}(n=1520) 28.7, p=0.001\right)$ as a contraceptive method. However, a similar proportion of women and men would consider the use of DMPA (women $13.4 \%$, men $4.9 \%$; $x^{2}(n=1520)$ 10.7, $p=0.001)$.

When the respondents were asked to give their opinion on the use of LARC, 98 (6.4\%) of them stated that they (or their partner) were currently using an intrauterine method, while 22 (1.4\%) respondents stated that they (or their partner) were currently using DMPA. Additionally, 404 (26.6\%) respondents reported that they had used intrauterine contraception. Finally, 98 (6.4\%) respondents declared that they were already familiar with DMPA. 
Table 1. Sociodemographic and reproductive history characteristics of women and men attending health clinics in the South East of Tehran, Iran $(n=1520)$

\begin{tabular}{|c|c|c|c|c|c|}
\hline Variables & $\begin{array}{c}\text { Women Frequency } \\
\text { Mean+/-SD }\end{array}$ & $\begin{array}{c}\text { Women Percentage } \\
\text { Mean+/-SD }\end{array}$ & $\begin{array}{c}\text { Men } \\
\text { Frequency Mean+/-SD } \\
\end{array}$ & $\begin{array}{c}\text { Men } \\
\text { Percentage Mean+/-SD } \\
\end{array}$ & Total \\
\hline Total & 1013 & 66.6 & 507 & 33.4 & 1520 \\
\hline Age & Mean+/-SD & Mean+/-SD & Mean+/-SD & Mean+/-SD & \\
\hline Years & $32.4 \pm 7.3$ & & $36.5 \pm 6.1$ & & $33.7 \pm 7.1$ \\
\hline Range & $16-49$ & & $23-49$ & & $16-49$ \\
\hline Age & Frequency & Percentage & Frequency & Percentage & \\
\hline $15-25 y$ & 117 & 95.7 & 8 & 4.3 & 185 \\
\hline $26-40 y$ & 696 & 65.5 & 366 & 34.5 & 1062 \\
\hline$>40 y$ & 140 & 51.3 & 133 & 48.7 & 273 \\
\hline \multicolumn{6}{|l|}{ Education } \\
\hline Diploma and below & 847 & 62.2 & 414 & 32.8 & 1261 \\
\hline Beyond diploma & 166 & 64.1 & 93 & 35.9 & 259 \\
\hline \multicolumn{6}{|l|}{ Employed } \\
\hline No & 946 & 99.7 & 3 & 0.3 & 949 \\
\hline Yes & 67 & 11.7 & 504 & 88.3 & 571 \\
\hline \multicolumn{6}{|l|}{ marriage } \\
\hline Once & 984 & 67.6 & 471 & 32.4 & 1455 \\
\hline More than once & 29 & 44.6 & 36 & 55.4 & 65 \\
\hline \multicolumn{6}{|l|}{ Partner 's age category } \\
\hline $18-25 y$ & 34 & 29.8 & 80 & 70.2 & 114 \\
\hline $26-40 y$ & 667 & 63.2 & 388 & 36.8 & 1055 \\
\hline$>40 y$ & 312 & 88.9 & 39 & 11.1 & 351 \\
\hline \multicolumn{6}{|l|}{ Partner's education } \\
\hline Diploma and below & 822 & 69.1 & 368 & 30.9 & 1190 \\
\hline Beyond diploma & 191 & 57.9 & 139 & 42.1 & 330 \\
\hline \multicolumn{6}{|l|}{ Religion } \\
\hline Shia Islam & 949 & 66.1 & 487 & 33.9 & 1436 \\
\hline Sunni Islam & 64 & 76.2 & 20 & 2.8 & 84 \\
\hline \multicolumn{6}{|l|}{ Important of religion in fertility } \\
\hline Unimportant & 243 & 69.6 & 106 & 30.4 & 349 \\
\hline Important & 770 & 65.8 & 401 & 34.2 & 1171 \\
\hline \multicolumn{6}{|l|}{ Satisfaction with finance status } \\
\hline Poor & 871 & 65.8 & 453 & 34.2 & 1324 \\
\hline good & 142 & 72.4 & & 27.6 & 196 \\
\hline satisfaction with general heal & & & & & \\
\hline Not good & 843 & 69 & 378 & 31 & 1221 \\
\hline Good & 170 & 56.9 & 129 & 43.1 & 299 \\
\hline Fertility knowledge & & & & & \\
\hline Poor & 700 & 73.6 & 251 & 26.4 & 951 \\
\hline Good & 29 & 74.4 & 10 & 25.6 & 39 \\
\hline History of pregnancy & & & & & \\
\hline No & 16 & 39 & 25 & 61 & 41 \\
\hline Yes & 997 & 67.4 & 482 & 32.6 & 1479 \\
\hline History of unwanted pregnan & & & & & \\
\hline None & 565 & 63.9 & 319 & 36.1 & 884 \\
\hline At least once & 448 & 70.4 & 188 & 37.1 & 636 \\
\hline Current contraception use & & & & & \\
\hline No & 173 & 74.89 & 58 & 25.1 & 231 \\
\hline Yes & 840 & 65.16 & 449 & 34.83 & 1289 \\
\hline Current contraception metho & & & & & \\
\hline Withdrawal, natural method & 352 & 65.2 & 188 & 34.8 & 540 \\
\hline Condom & 238 & 57.1 & 179 & 42.9 & 417 \\
\hline Oral contraceptive pill & 72 & 79.1 & 19 & 20.9 & 91 \\
\hline Contraception device IUD & 98 & 78.4 & 27 & 21.6 & 125 \\
\hline Contraception injection DMPA & 22 & 84.6 & 4 & 15.4 & 26 \\
\hline TL & 45 & 4.4 & 22 & 4.3 & 67 \\
\hline VS & 13 & 56.5 & 10 & 43.5 & 23 \\
\hline None of these conditions & 173 & 74.9 & 58 & 25.1 & 231 \\
\hline $\begin{array}{l}\text { Health consultation for contr } \\
\text { past } 2 \text { years }\end{array}$ & & & & & \\
\hline No & 444 & 49 & 462 & 51 & 906 \\
\hline Yes & 569 & 92.7 & 45 & 7.3 & 614 \\
\hline Comfortable asking health ca & & & & & \\
\hline Uncomfortable & 233 & 47.5 & 258 & 52.5 & 491 \\
\hline Comfortable & 780 & 75.8 & 249 & 24.2 & 1029 \\
\hline History of abortion & & & & & \\
\hline No & 918 & 66.3 & 466 & 33.7 & 1384 \\
\hline Yes & 95 & 69.9 & 41 & 30.1 & 136 \\
\hline Importance of avoiding pregn & & & & & \\
\hline Not at all important & 10 & 37 & 17 & 63 & 27 \\
\hline Very important & 1003 & 67.2 & 490 & 32.8 & 1493 \\
\hline Comfortable talking with part & & & & & \\
\hline Uncomfortable & 127 & 76 & 40 & 24 & 167 \\
\hline Comfortable & 886 & 65.5 & 467 & 34.5 & 1353 \\
\hline Comfortable asking about avo & & & & & \\
\hline Uncomfortable & 129 & 75.9 & 41 & 24.1 & 170 \\
\hline Comfortable & 884 & 65.5 & 466 & 34.5 & 1350 \\
\hline
\end{tabular}


Table 2. Comparison of responses by women and men attending health clinics in South East Tehran, Iran (N=1520)

\begin{tabular}{|c|c|c|c|c|c|c|}
\hline Variables & $\begin{array}{l}\text { Women } \\
\mathrm{N}=1013 \\
\end{array}$ & $\begin{array}{c}\text { Women } \\
\text { Percentage }\end{array}$ & $\begin{array}{c}\text { Men } \\
\mathrm{N}=507\end{array}$ & $\begin{array}{c}\text { Men } \\
\text { Percentage } \\
\end{array}$ & Total & $\begin{array}{c}\text { p- } \\
\text { value }\end{array}$ \\
\hline Heard of this method (IUD) & & & & & & 0.001 \\
\hline Yes & 933 & 72.3 & 358 & 27.7 & 1291 & \\
\hline No & 77 & 34.5 & 146 & 65.5 & 223 & \\
\hline I don't know & 3 & 50 & 3 & 50 & 6 & \\
\hline Method (IUD) is reliable & & & & & & 0.001 \\
\hline Yes & 349 & 76.9 & 105 & 23.1 & 454 & \\
\hline No & 580 & 68 & 273 & 32 & 853 & \\
\hline I don't know & 84 & 39.4 & 129 & 60.6 & 213 & \\
\hline $\begin{array}{l}\text { Would consider using this } \\
\text { method (IUD) }\end{array}$ & & & & & & 0.001 \\
\hline Yes & 331 & 75.9 & 105 & 24.1 & 436 & \\
\hline No & 611 & 69.3 & 271 & 30.7 & 882 & \\
\hline I don't know & 71 & 35.1 & 131 & 64.9 & 202 & \\
\hline Heard of this method (DMPA) & & & & & & 0.001 \\
\hline Yes & 750 & 79.7 & 191 & 20.3 & 941 & \\
\hline No & 260 & 45.5 & 311 & 54.5 & 571 & \\
\hline I don't know & 3 & 37.5 & 5 & 62.5 & 8 & \\
\hline Method (DMPA) is reliable & & & & & & 0.001 \\
\hline Yes & 156 & 84.3 & 29 & 15.7 & 185 & \\
\hline No & 599 & 74.8 & 202 & 25.2 & 801 & \\
\hline I don't know & 258 & 48.3 & 276 & 51.7 & 534 & \\
\hline $\begin{array}{l}\text { Would consider using this } \\
\text { method (DMPA) }\end{array}$ & & & & & & 0.001 \\
\hline Yes & 136 & 84.5 & 25 & 15.5 & 161 & \\
\hline No & 623 & 75.3 & 204 & 24.7 & 827 & \\
\hline I don't know & 254 & 47.7 & 278 & 52.3 & 532 & \\
\hline
\end{tabular}

\section{Factors Associated with the Perceived Reliability and Consideration of Long-acting Methods}

Perceived reliability of IUD among_women was found to be significantly associated with age $(p=0.001)$, level of education $(p=0.001)$, the age of their partner $(p=0.001)$, religion $(p=0.018)$, overall health satisfaction $(p=0.035)$, history of pregnancy $(p=0.001)$, unwanted pregnancy $(p=0.001)$ and abortion ( $p=0.023)$, and current contraception use $(p=0.003)$.

Associated factors with the reliability of IUDs in men were as follows: age of men $(p=0.006)$, level of participant's education $(p=0.004)$ and that of his spouse $(p=0.001)$, history of unwanted pregnancy $(p=0.003)$ and abortion $(p=0.025)$, consulting with health professionals $(p=0.034)$, and current contraception use $(p=0.015)$ (Table 3$)$.

The perceived reliability of DMPA among women was significantly associated with education $(p=0.003)$, partner's education $(p=0.053)$, religion $(p=0.055)$, financial satisfaction $(p=0.001)$, overall health satisfaction $(p=0.001)$, history of unwanted pregnancy $(p=0.008)$ and current contraception use $(p=0.003)$. Meanwhile, the perceived unreliability of DMPA among men was only associated with multiple marriages $(p=0.003)$ (Table 3).

Willingness to consider using IUD by women was significantly associated with the following variables: age ( $p=0.001)$, education $(p=0.001)$, the age of their partner ( $p=0.001)$, partner's education $(p=0.001)$, history of pregnancy $(p=0.001)$, unwanted pregnancy $(p=0.001)$ and current contraception method ( $p=0.010)$. Meanwhile, the use of IUDs in men was only associated with age $(p=0.001)$, level of education $(p=0.001)$, partner's education $(p=0.001)$, history of pregnancy $(p=0.001)$, history of unwanted pregnancy ( $p=0.034)$, consulting with health professionals $(p=0.013)$ and current contraception method $(p=0.023)$ (Table 4).

Willingness to consider using DMPA by women was significantly associated with the following variables: level of
Table 3. Univariable associations with respondent characteristics and perception of IUDs as reliable $(n=1520)$

\begin{tabular}{|c|c|c|c|c|c|}
\hline \multirow{2}{*}{$\begin{array}{l}\text { Characteristics } \\
\text { WOMEN }\end{array}$} & \multicolumn{5}{|c|}{ Consider IUDs reliable (\%) } \\
\hline & Yes & No & $\begin{array}{l}\text { I don't } \\
\text { know }\end{array}$ & $\chi^{2}$ & $\begin{array}{c}\text { p- } \\
\text { value }\end{array}$ \\
\hline Age $(\mathrm{N}=1013)$ & & & & 42.68 & 0.001 \\
\hline $15-25 y(n=177)$ & 17.5 & 68.9 & 13.6 & & \\
\hline $26-40 y(n=696)$ & 35.6 & 57.2 & 7.2 & & \\
\hline$>40 \mathrm{y}(\mathrm{n}=140)$ & 50 & 42.9 & 7.1 & & \\
\hline Education (N=1013) & & & & 10.23 & 0.001 \\
\hline Diploma and below $(\mathrm{n}=847)$ & 36.5 & 55.3 & 8.3 & & \\
\hline beyond diploma $(\mathrm{n}=166)$ & 24.1 & 67.5 & 8.4 & & \\
\hline Partner's age (N=1013) & & & & 64.98 & 0.001 \\
\hline $18-25 y(n=34)$ & 14.7 & 73.5 & 11.8 & & \\
\hline $26-40 y(n=667)$ & 27.1 & 64 & 8.8 & & \\
\hline$>40 \mathrm{y}(\mathrm{n}=312)$ & 52.2 & 41 & 6.7 & & \\
\hline Religion (N=1013) & & & & 7.98 & 0.018 \\
\hline Shia Islam $(n=949)$ & 33.4 & 58.4 & 8.2 & & \\
\hline Sunni Islam $(\mathrm{n}=67)$ & 50 & 40.6 & 9.4 & & \\
\hline Satisfaction with general health status(N=1013) & & & & 6.83 & 0.035 \\
\hline Not good $(n=843)$ & 36.1 & 56.2 & 7.7 & & \\
\hline Good $(n=170)$ & 26.5 & 62.4 & 11.2 & & \\
\hline History of pregnancy $(\mathrm{N}=1013)$ & & & & 31.52 & 0.001 \\
\hline No $(n=13)$ & 0 & 43.8 & 56.3 & & \\
\hline Yes $(\mathrm{n}=997)$ & 35 & 57.5 & 7.5 & & \\
\hline History of unwanted pregnancy ( $\mathrm{N}=1013)$ & & & & 30.91 & 0.001 \\
\hline None $(n=565)$ & 27.1 & 63.4 & 9.6 & & \\
\hline At least once $(\mathrm{n}=448)$ & 43.6 & 49.6 & 6.7 & & \\
\hline Current contraception use(N=1013) & & & & 11.35 & 0.003 \\
\hline No $(n=173)$ & 29.5 & 55.5 & 15 & & \\
\hline Yes $(n=840)$ & 35.5 & 57.6 & 6.9 & & \\
\hline
\end{tabular}

contraception, pregnancy or fertility over t

past 2 years $(\mathrm{N}=1013)$

past 2 years $(\mathrm{N}=1013)$ \begin{tabular}{lccc} 
No $(\mathrm{n}=444)$ & 33.6 & 55.9 & 10.69 \\
\hline Yes $(\mathrm{n}=569)$ & 35.1 & 58.3 & 6.59
\end{tabular}

\begin{tabular}{llll}
\hline History of abortion $(\mathrm{N}=1013)$ & 7.80 & 0.023 \\
\hline
\end{tabular}

\begin{tabular}{llllll}
\hline No $(n=839)$ & 33.1 & 58.3 & 8.6 & &
\end{tabular}

\begin{tabular}{llll} 
Yes $(\mathrm{n}=95)$ & 47.4 & 47.4 & 5.3 \\
\hline MEN & & &
\end{tabular}

\begin{tabular}{lll}
\hline Age (N=507) & 14.60 & 0.006
\end{tabular}

\begin{tabular}{llll}
\hline $15-25 \mathrm{y}(\mathrm{n}=8)$ & 12.5 & 62.5 & 25 \\
\hline $26-40 \mathrm{y}(\mathrm{n}=366)$ & 16.7 & 55.7 & 27.6
\end{tabular}

\begin{tabular}{lrrr}
\hline $26-40 \mathrm{y}(\mathrm{n}=366)$ & 16.7 & 55.7 & 27.6 \\
\hline & 32.3 & 48.1 & 19.5
\end{tabular}

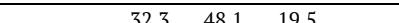

\begin{tabular}{llll}
\hline Education $(\mathrm{N}=507)$ & 11.19 & 0.004 \\
\hline
\end{tabular}

\begin{tabular}{lllll}
\hline Diploma and below $(\mathrm{n}=414)$ & 22.7 & 50.5 & 26.8 & \\
\hline
\end{tabular}

\begin{tabular}{llll}
\hline Beyond diploma $(\mathrm{n}=94)$ & 11.8 & 68.8 & 19.4 \\
\hline
\end{tabular}

\begin{tabular}{llll}
\hline Partner's age $(\mathbf{N}=\mathbf{5 0 7})$ & 8.29 & 0.082 \\
\hline
\end{tabular}

$\begin{array}{llll}18-25 \mathrm{y}(\mathrm{n}=80) & 11.3 & 55 & 33.8\end{array}$

\begin{tabular}{lccc}
\hline $26-40 \mathrm{y}(\mathrm{n}=388)$ & 21.9 & 54.4 & 23.7 \\
\hline
\end{tabular}

$\begin{array}{llll}>40 \mathrm{y}(\mathrm{n}=39) & 28.3 & 46.2 & 25.6\end{array}$

\begin{tabular}{llll}
\hline Partner's education(N=507) & 16.06 & 0.001 \\
\hline
\end{tabular}

\begin{tabular}{lllllll}
\hline Diploma and below $(\mathrm{n}=368)$ & 23.9 & 48.6 & 27.4 & & & \\
\hline
\end{tabular}

\begin{tabular}{llll} 
Beyond Diploma $(\mathrm{n}=139)$ & 12.2 & 67.6 & 20.1 \\
\hline
\end{tabular}

\begin{tabular}{llll}
\hline History of unwanted pregnancy $(\mathrm{N}=507)$ & 11.39 & 0.003 \\
\hline
\end{tabular}

\begin{tabular}{llll}
\hline None $(\mathrm{n}=322)$ & 16.3 & 55.2 & 28.5 \\
\hline
\end{tabular}

\begin{tabular}{llll}
\hline At least once $(\mathrm{n}=185)$ & 28.2 & 51.6 & 20.2 \\
\hline
\end{tabular}

Had a consult with health professionals for

contraception, pregnancy or fertility over the

past 2 years $(\mathrm{N}=507)$

No $(\mathrm{n}=462)$

\begin{tabular}{llllll}
\hline & 8.9 & 53.3 & 37.8 & & \\
\hline History of abortion $(\mathbf{N}=\mathbf{5 0 7})$ & & & & 7.35 & 0.025 \\
\hline
\end{tabular}

\begin{tabular}{lllllll}
\hline No $(\mathrm{n}=458)$ & 20 & 53.2 & 28.8 & & & \\
\hline$Y$
\end{tabular}

\begin{tabular}{llll}
\hline Yes $(\mathrm{n}=49)$ & 29.3 & 61 & 9.8 \\
\hline
\end{tabular}

\begin{tabular}{lllll}
\hline Current contraception use (N=507) & 8.35 & 0.015 \\
\hline
\end{tabular}

\begin{tabular}{llll}
\hline No $(\mathrm{n}=58)$ & 13.8 & 44.8 & 41.4 \\
\hline
\end{tabular}

Yes $(\mathrm{n}=449)$

All data are presented as row percent.

$P$-values are based on c2 test for comparison of categorical variables between conside

intrauterine contraception reliable and do not consider intrauterine contraception reliable

education ( $p=0.001)$, the age of their partner $(p=0.033)$, their partner's education $(p=0.003)$, religion $(p=0.034)$, history of pregnancy $(p=0.007)$, history of unwanted pregnancy $(p=0.001)$, level of comfort talking to a health care provider about contraception $(p=0.009)$, and current contraception method ( $p=0.003$ ) (Table 5). The male participants regarded marriage $(p=0.011)$ and the current contraception method $(p=0.041)$ as important factors that influenced fertility choices. 
Table 4. Univariable associations with respondent characteristics and perception of DMPA as reliable_( $\mathrm{n}=1520)$

\begin{tabular}{|c|c|c|c|c|c|}
\hline \multirow{2}{*}{$\begin{array}{l}\text { Characteristics } \\
\text { WOMEN }\end{array}$} & \multicolumn{5}{|c|}{ Consider DMPA reliable (\%) } \\
\hline & Yes & No & $\begin{array}{l}\text { I don't } \\
\text { know }\end{array}$ & $\chi^{2}$ & $\begin{array}{c}\mathbf{p -} \\
\text { value }\end{array}$ \\
\hline Education ( N=1013) & & & & 11.45 & 0.003 \\
\hline Diploma and below $(n=847)$ & 16.9 & 57.4 & 25.7 & & \\
\hline beyond diploma $(\mathrm{n}=166)$ & 7.8 & 68.1 & 24.1 & & \\
\hline Partner's age $(\mathrm{N}=1013)$ & & & & 4.74 & 0.315 \\
\hline $18-25 y(n=34)$ & 14.7 & 64.7 & 20.6 & & \\
\hline $26-40 y(n=667)$ & 14.2 & 58.5 & 27.3 & & \\
\hline$>40 \mathrm{y}(\mathrm{n}=312)$ & 17.9 & 59.5 & 22.1 & & \\
\hline Partner's education $(\mathrm{N}=1013)$ & & & & 5.87 & 0.053 \\
\hline Diploma and below $(\mathrm{n}=822)$ & 16.7 & 58.3 & 25.1 & & \\
\hline Beyond diploma $(\mathrm{n}=191)$ & 9.9 & 62.8 & 27.2 & & \\
\hline Religion (N=1013) & & & & 5.79 & 0.055 \\
\hline Shia Islam $(\mathrm{n}=949)$ & 14.6 & 59.5 & 25.8 & & \\
\hline Sunni Islam $(n=64)$ & 26.6 & 53.1 & 20.3 & & \\
\hline Satisfaction with finance status (N=1013) & & & & 306.80 & 0.001 \\
\hline Poor $(n=871)$ & 15.3 & 59.6 & 25.1 & & \\
\hline Good $(n=142)$ & 16.2 & 56.3 & 27.5 & & \\
\hline Satisfaction with general health status $(\mathrm{N}=1013)$ & & & & 24.60 & 0.001 \\
\hline Not Good $(n=843)$ & 15.8 & 59.1 & 24.9 & & \\
\hline $\operatorname{good}(n=170)$ & 13.5 & 58.2 & 25.5 & & \\
\hline History of unwanted pregnancy $(\mathrm{N}=1013)$ & & & & 9.64 & 0.008 \\
\hline None $(n=565)$ & 12.4 & 60.2 & 27.4 & & \\
\hline At least once $(n=448)$ & 19.2 & 57.8 & 23 & & \\
\hline Current contraception use $(\mathrm{N}=1013)$ & & & & 11.422 & 0.003 \\
\hline No $(n=173)$ & 16.2 & 48.6 & 35.3 & & \\
\hline Yes $(n=840)$ & 15.2 & 61.3 & 23.5 & & \\
\hline \multicolumn{6}{|l|}{ MEN } \\
\hline Marriage (N=507) & & & & 11.39 & 0.003 \\
\hline Once $(n=471)$ & 5.9 & 37.8 & 36.3 & & \\
\hline More than once $(\mathrm{n}=36)$ & 2.8 & 66.7 & 30.6 & & \\
\hline
\end{tabular}

Women who did not consider IUDs to be a reliable method were more likely to avoid contraception and to have never been pregnant. Additionally, they were Shia Muslims, had just married once, and their partners were 18-40 years old.

Additionally, women who did not have enough information about IUDs reliability (women who answered "I don't know" in the questionnaire) did not undergo any contraception, and the age of their partners varied from 26-40 years old. Those women had not consulted with health professionals about contraception over the past two years. Additionally, men's partners did not have any history of unwanted pregnancy or contraception, and their education level was 'Diploma or below.'

Women who did not consider DMPA a reliable method did not have any history of unwanted pregnancy and their education level was 'Diploma or below'.

Moreover, women who did not have enough information about DMPAs reliability (women who answered "I don't know" in the questionnaire) did not have any history of unwanted pregnancy, and they were all Shia Muslims.

Women who were more likely not to consider the use of

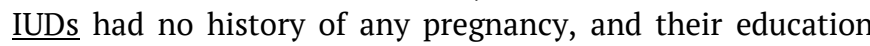
level was 'Diploma or below'. The age of their partners was between 18-25 years old. In addition, they were not using contraception at the moment. Men who responded that they would not consider using IUDs reported that their partners did not have any history of pregnancy and the age of their partners varied from 26-40 years old. Their education level was 'Diploma or below'.

Women who did not have any history of unwanted pregnancy and the age of their partners varied from 26-40
Table 5. Univariable associations between respondent characteristics and consideration of use of IUDs $(n=1520)$

\begin{tabular}{|c|c|c|c|c|c|}
\hline \multirow{2}{*}{$\begin{array}{l}\text { Characteristics } \\
\text { WOMEN }\end{array}$} & \multicolumn{5}{|c|}{ Consider using IUDs (\%) } \\
\hline & Yes & No & $\begin{array}{l}\text { I don't } \\
\text { know }\end{array}$ & $\chi^{2}$ & $\begin{array}{c}\mathbf{p}- \\
\text { value }\end{array}$ \\
\hline Age (N=1013) & & & & 41.73 & 0.001 \\
\hline $15-25 y(n=177)$ & 16.9 & 72.9 & 10.2 & & \\
\hline $26-40 y(n=696)$ & 33.2 & 60.5 & 6.3 & & \\
\hline$>40 \mathrm{y}(\mathrm{n}=140)$ & 50 & 43.6 & 6.4 & & \\
\hline Education $(\mathrm{N}=1013)$ & & & & 31.79 & 0.001 \\
\hline Diploma and below $(n=846)$ & 35.9 & 56.7 & 7.4 & & \\
\hline Beyond diploma $(\mathrm{n}=166)$ & 16.3 & 78.9 & 4.8 & & \\
\hline Partner's age $(\mathrm{N}=1013)$ & & & & 65.98 & 0.001 \\
\hline $18-25 \mathrm{y}(\mathrm{n}=34)$ & 14.7 & 70.6 & 14.7 & & \\
\hline $26-40 y(n=667)$ & 25.3 & 67.5 & 7.2 & & \\
\hline$>40 \mathrm{y}(\mathrm{n}=312)$ & 50.3 & 43.9 & 5.8 & & \\
\hline Partner's education $(\mathrm{N}=1013)$ & & & & 15.63 & 0.001 \\
\hline Diploma and below $(\mathrm{n}=822)$ & 34.7 & 57.5 & 7.8 & & \\
\hline Beyond diploma $(\mathrm{n}=191)$ & 24.1 & 72.3 & 3.7 & & \\
\hline History of pregnancy $(\mathrm{N}=1013)$ & & & & 15.65 & 0.001 \\
\hline No $(n=16)$ & 0 & 62.5 & 37.5 & & \\
\hline Yes $(n=997)$ & 33.2 & 60.3 & 6.5 & & \\
\hline History of unwanted pregnancy $(\mathrm{N}=1013)$ & & & & 21.30 & 0.001 \\
\hline None $(n=665)$ & 25.1 & 66.2 & 8.7 & & \\
\hline At least once $(n=448)$ & 42.2 & 52.9 & 4.9 & & \\
\hline Current contraception use $(\mathrm{N}=1013)$ & & & & 9.13 & 0.010 \\
\hline No $(n=173)$ & 29.5 & 57.8 & 12.7 & & \\
\hline Yes $(n=840)$ & 33.5 & 60.7 & 5.8 & & \\
\hline \multicolumn{6}{|l|}{ MEN } \\
\hline Age $(\mathrm{N}=507)$ & & & & 14.26 & 0.006 \\
\hline $15-25 \mathrm{y}(\mathrm{n}=8)$ & 25 & 50 & 25 & & \\
\hline $26-40 y(n=366)$ & 16.4 & 56.3 & 27.3 & & \\
\hline$>40 \mathrm{y}(\mathrm{n}=133)$ & 32.2 & 45.9 & 21.8 & & \\
\hline Education (N=507) & & & & 13.07 & 0.001 \\
\hline Diploma and below $(\mathrm{n}=414)$ & 22.7 & 49.8 & 27.5 & & \\
\hline Beyond diploma $(\mathrm{n}=93)$ & 11.8 & 68.9 & 18.3 & & \\
\hline Partner's age $(\mathrm{N}=507)$ & & & & 6.72 & 0.156 \\
\hline $18-25 y(n=80)$ & 12.5 & 55 & 32.5 & & \\
\hline $26-40 y(n=388)$ & 21.6 & 54.1 & 24.2 & & \\
\hline$>40 \mathrm{y}(\mathrm{n}=39)$ & 28.2 & 43.6 & 28.2 & & \\
\hline Partner's education $(\mathrm{N}=507)$ & & & & 19.90 & 0.001 \\
\hline Diploma and below $(\mathrm{n}=368)$ & 24.7 & 48.1 & 27.2 & & \\
\hline Beyond diploma $(\mathrm{n}=139)$ & 10.1 & 67.6 & 22.3 & & \\
\hline History of pregnancy $(\mathrm{N}=507)$ & & & & 13.44 & 0.001 \\
\hline No $(n=25)$ & 0 & 56 & 44 & & \\
\hline Yes $(n=482)$ & 21.8 & 53.3 & 24.9 & & \\
\hline History of unwanted pregnancy $(\mathrm{N}=507)$ & & & & 6.96 & 0.034 \\
\hline None $(\mathrm{n}=319)$ & 17.2 & 54.5 & 28.2 & & \\
\hline At least once $(n=188)$ & 26.6 & 51.6 & 21.8 & & \\
\hline $\begin{array}{l}\text { Had a consult with health professionals for } \\
\text { contraception, pregnancy or fertility over the } \\
\text { past } 2 \text { years }(\mathrm{N}=507)\end{array}$ & & & & 8.74 & 0.013 \\
\hline No $(n=462)$ & 21.9 & 53.9 & 24.2 & & \\
\hline Yes $(n=45)$ & 8.9 & 48.9 & 42.2 & & \\
\hline Current contraception use $(\mathrm{N}=507)$ & & & & 7.62 & 0.023 \\
\hline No $(n=58)$ & 17.2 & 41.4 & 41.4 & & \\
\hline Yes $(n=449)$ & 21.2 & 55 & 23.8 & & \\
\hline
\end{tabular}

Yes $(\mathrm{n}=449)$

All data are presented as row percent.

$P$-values are based on $\mathrm{c} 2$ test for comparison of categorical variables between would consider use of intrauterine contraception and would not consider use of intrauterine contraception

years old reported that they had no information about using IUDs (They answered “I don't know in the questionnaire).

Women who did not consider using DMPA had not used any contraception at the time when they were filling out the questionnaire; their ages were between 26-40 years old.

Women who did not have enough information about using DMPA (They answered "I don't know in the questionnaire) were not comfortable enough to ask their health care providers about contraception, compared to those who insisted on using DMPA. 
Table 6. Univariable associations with respondent characteristics and consideration of use of DMPA $(n=1520)$

\begin{tabular}{|c|c|c|c|c|c|}
\hline \multirow{2}{*}{$\begin{array}{l}\text { Characteristics } \\
\text { WOMEN }\end{array}$} & \multicolumn{5}{|c|}{ Consider using DMPA (\%) } \\
\hline & Yes & No & $\begin{array}{l}\text { I don't } \\
\text { know }\end{array}$ & $\chi^{2}$ & $\begin{array}{c}\mathbf{p}- \\
\text { value }\end{array}$ \\
\hline Education (N=1013) & & & & 17.23 & 0.001 \\
\hline Diploma and below $(n=847)$ & 15.2 & 59 & 25.7 & & \\
\hline Beyond diploma $(\mathrm{n}=166)$ & 5.4 & 72.9 & 21.7 & & \\
\hline Partner's age $(\mathrm{N}=1013)$ & & & & 10.62 & 0.033 \\
\hline $18-25 y(n=34)$ & 5.9 & 76.5 & 17.6 & & \\
\hline $26-40 y(n=599)$ & 12 & 61.5 & 26.5 & & \\
\hline$>40 \mathrm{y}(\mathrm{n}=312)$ & 17.9 & 59.3 & 22.8 & & \\
\hline Partner's education $(\mathrm{N}=1013)$ & & & & 11.81 & 0.003 \\
\hline Diploma and below $(\mathrm{n}=822)$ & 15.2 & 59.5 & 25.3 & & \\
\hline Beyond diploma $(\mathrm{n}=191)$ & 6.8 & 69.1 & 24.) & & \\
\hline Religion (N=1013) & & & & 6.76 & 0.034 \\
\hline Shia Islam $(\mathrm{n}=949)$ & 12.9 & 61.6 & 25.5 & & \\
\hline Sunni Islam(n=64) & 25 & 56.3 & 18.8 & & \\
\hline Important of religion in fertility $(\mathrm{N}=1013)$ & & & & 5.26 & 0.072 \\
\hline Unimportant $(n=243)$ & 16.5 & 63.5 & 20.2 & & \\
\hline Important $(\mathrm{n}=770)$ & 12.7 & 60.6 & 26.6 & & \\
\hline History of pregnancy $(\mathrm{N}=1013)$ & & & & 9.96 & 0.007 \\
\hline No $(n=16)$ & 0 & 43.8 & 56.2 & & \\
\hline Yes $(\mathrm{n}=997)$ & 13.8 & 61.6 & 24.6 & & \\
\hline History of unwanted pregnancy $(\mathrm{N}=1013)$ & & & & 15.75 & 0.001 \\
\hline None $(n=565)$ & 9.9 & 62.8 & 27.3 & & \\
\hline At least once $(n=448)$ & 18.3 & 59.4 & 22.3 & & \\
\hline $\begin{array}{l}\text { How comfortable are you asking your health } \\
\text { care provider about contraception matters? } \\
(\mathrm{N}=1013)\end{array}$ & & & & 9.13 & 0.009 \\
\hline Uncomfortable $(\mathrm{n}=233)$ & 17.6 & 63.9 & 18.5 & & \\
\hline Comfortable $(\mathrm{n}=780)$ & 12.4 & 60.5 & 27.1 & & \\
\hline Current contraception use $(\mathrm{N}=1013)$ & & & & 11.67 & 0.003 \\
\hline No $(n=173)$ & 15 & 50.3 & 34.7 & & \\
\hline Yes $(n=840)$ & 13.3 & 63.6 & 23.1 & & \\
\hline MEN & & & & & \\
\hline Marriage (N=507) & & & & 8.82 & 0.011 \\
\hline Once $(n=471)$ & 5.1 & 38.4 & 56.5 & & \\
\hline More than once $(n=36)$ & 2.8 & 63.9 & 33.3 & & \\
\hline Current contraception use $(\mathrm{N}=507)$ & & & & 6.43 & 0.041 \\
\hline No $(n=58)$ & 0 & 39.7 & 60.3 & & \\
\hline Yes $(n=449)$ & 5.6 & 40.3 & 54.1 & & \\
\hline
\end{tabular}

Table 7. Adjusted model of factors associated with perception of LARC as reliable

\begin{tabular}{|c|c|c|c|}
\hline Independent variable & $\begin{array}{c}\text { p- } \\
\text { value }\end{array}$ & $\begin{array}{c}\text { Adjusted } \\
\text { odds ratio }\end{array}$ & $95 \% \mathrm{CI}$ \\
\hline \multicolumn{4}{|l|}{ IUD Women $(\mathrm{N}=1013)$} \\
\hline \multicolumn{4}{|l|}{ IUD Reliable $=$ NO } \\
\hline Religion (Shia Islam) & 0.001 & 2.69 & $1.51-4.79$ \\
\hline History of unwanted pregnancy (NO) & 0.001 & 1.64 & $1.23-2.19$ \\
\hline Partner's age $18-25 y$ & 0.006 & 4.69 & $1.57-13.97$ \\
\hline Partner's age $26-40 \mathrm{y}$ & 0.001 & 2.62 & $1.82-3.77$ \\
\hline \multicolumn{4}{|l|}{ IUD Reliable = I don't know } \\
\hline Current contraception use (NO) & 0.001 & 2.59 & $1.46-4.62$ \\
\hline Partner 's age category (26-40y) & 0.03 & 2.14 & $1.05-4.37$ \\
\hline History of unwanted pregnancy (NO) & 0.04 & 1.72 & $1.02-2.88$ \\
\hline $\begin{array}{l}\text { Had a consult with health professionals for contraception, } \\
\text { pregnancy or fertility over the past } 2 \text { years (No) }\end{array}$ & 0.002 & 2.29 & $1.37-3.83$ \\
\hline \multicolumn{4}{|l|}{ IUD Men $(\mathrm{N}=507)$} \\
\hline \multicolumn{4}{|l|}{ IUD Reliable $=$ No } \\
\hline History of unwanted pregnancy (NO) & 0.01 & 1.85 & $1.12-3.05$ \\
\hline Age( 26-40Y) & 0.03 & 1.81 & $1.03-3.17$ \\
\hline Education (Diploma and below) & 0.02 & 0.44 & $0.22-0.89$ \\
\hline \multicolumn{4}{|l|}{ IUD Reliable = I don't know } \\
\hline History of unwanted pregnancy (NO) & 0.01 & 2.08 & $1.16-3.73$ \\
\hline Current contraception use (NO) & 0.04 & 2.43 & $1.19-4.96$ \\
\hline Education (Beyond diploma) & 0.03 & 1.81 & $1.04-3.17$ \\
\hline \multicolumn{4}{|l|}{ DMPA Women (n=1013) } \\
\hline \multicolumn{4}{|l|}{ DMPA Reliable $=$ NO } \\
\hline History of unwanted pregnancy (NO) & 0.02 & 1.54 & $1.07-2.24$ \\
\hline Education (Beyond diploma) & 0.07 & 0.43 & $0.23-0.79$ \\
\hline \multicolumn{4}{|l|}{ DMPA Reliable = I don't know } \\
\hline History of unwanted pregnancy (NO) & 0.02 & 1.62 & $1.07-2.47$ \\
\hline Religion ( Shia Islam) & 0.03 & 2.25 & $1.05-4.87$ \\
\hline
\end{tabular}

Table 8. Adjusted model of factors associated with consideration of use of LARC

\begin{tabular}{|c|c|c|c|}
\hline Independent variable & $\begin{array}{c}\mathbf{p}- \\
\text { value }\end{array}$ & $\begin{array}{c}\text { Adjusted } \\
\text { odds ratio }\end{array}$ & $95 \%$ CI \\
\hline \multicolumn{4}{|l|}{ IUD Women $(\mathrm{n}=1013)$} \\
\hline \multicolumn{4}{|l|}{ Would/have consider(ed) IUD = NO } \\
\hline History of unwanted pregnancy (NO) & 0.03 & 1.45 & $1.02-2.04$ \\
\hline Education (Beyond diploma) & 0.001 & 0.37 & $0.2-0.66$ \\
\hline Age (18-25y) & 0.03 & 2.46 & $1.09-5.57$ \\
\hline Partner's age (26-40y) & 0.001 & 3.15 & $2.09-4.73$ \\
\hline \multicolumn{4}{|c|}{ Would/have consider(ed) IUD =I don't know } \\
\hline History of unwanted pregnancy (NO) & 0.01 & 2.08 & $1.13-3.83$ \\
\hline Current contraception use (NO) & 0.04 & 1.89 & $1.01-3.54$ \\
\hline Partner's age (26-40y) & 0.01 & 2.81 & $1.28-6.17$ \\
\hline \multicolumn{4}{|l|}{ IUD Men $(\mathrm{n}=507)$} \\
\hline \multicolumn{4}{|l|}{ Would/have consider(ed) IUD = NO } \\
\hline Age $(26-40 y)$ & 0.01 & 1.86 & $1.25-3.07$ \\
\hline Partner's education (Beyond diploma) & 0.01 & 0.41 & $0.21-0.82$ \\
\hline \multicolumn{4}{|c|}{ Would/have consider(ed) IUD =I don't know } \\
\hline Age (26-40y) & 0.02 & 1.97 & $1.09-3.54$ \\
\hline \multicolumn{4}{|l|}{ DMPA Women $(\mathrm{n}=1013)$} \\
\hline \multicolumn{4}{|l|}{ Would/have consider(ed ) DMPA $=$ NO } \\
\hline History of unwanted pregnancy (No) & 0.006 & 1.74 & $1.17-2.57$ \\
\hline Religion (Shia Islam) & 0.03 & 1.97 & $1.03-3.75$ \\
\hline Education (Beyond diploma) & 0.003 & 0.34 & $0.17-0.70$ \\
\hline \multicolumn{4}{|c|}{ Would/have consider(ed ) DMPA =I don't know } \\
\hline History of unwanted pregnancy (NO) & 0.007 & 1.83 & $1.18-2.84$ \\
\hline Religion (Shia Islam) & 0.01 & 2.79 & $1.24-6.25$ \\
\hline
\end{tabular}

Table 9. Summary of factors associated with LARC's perceived reliability and consideration of use

\begin{tabular}{|c|c|c|c|c|}
\hline & IUD & IUD & DMPA & DMPA \\
\hline Factors & $\begin{array}{c}\text { Perceive } \\
\text { d to be } \\
\text { reliable }\end{array}$ & $\begin{array}{c}\text { Would } \\
\text { consider } \\
\text { use }\end{array}$ & $\begin{array}{l}\text { Perceived } \\
\text { to be } \\
\text { reliable }\end{array}$ & $\begin{array}{c}\text { Would } \\
\text { consider } \\
\text { use }\end{array}$ \\
\hline Religion (Shia Islam) & $\mathrm{W}$ & & $\mathrm{W}$ & $\mathrm{W}$ \\
\hline Partner's age (18-25y) & W & & & \\
\hline Partner's age category (26-40y) & $\mathrm{W} / \mathrm{M}$ & $\mathrm{W}$ & & \\
\hline Current contraception use (NO) & W/M & $\mathrm{W}$ & & \\
\hline $\begin{array}{l}\text { Had consulted with health professionals about } \\
\text { contraception (NO) }\end{array}$ & $\mathrm{W}$ & & & \\
\hline History of unwanted pregnancy (NO) & $\mathrm{W} / \mathrm{M}$ & $\mathrm{W}$ & $\mathrm{W}$ & $\mathrm{W}$ \\
\hline Age (18-25y) & & $\mathrm{W}$ & & \\
\hline Age (26-40y) & $\mathrm{M}$ & $\mathrm{M}$ & & \\
\hline Education (Beyond diploma) & & $\mathrm{W}$ & $\mathrm{W}$ & $\mathrm{W}$ \\
\hline Partner's Education (Beyond diploma) & & $\mathrm{M}$ & & \\
\hline
\end{tabular}

\section{W, women; $M$, me}

\section{DISCUSSION}

The present study aimed to investigate a large sample size, which included the entire southern part and suburbs of Tehran, covering a population of over 1.5 million people with low socioeconomic status. Most of these areas suffered from high rates of unemployment, and inhabitants live in rented housing. These people are more likely to be at risk of unwanted pregnancy and need to pay more attention to their fertility status.

This research is the first of its kind, which aimed to investigate the perspectives of Iranian people about LARC. According to the findings, most Iranian men and women who are of reproductive age and are trying to avoid pregnancy are highly aware of LARC. However, LARC is not widely used among these couples.

\section{LARC Awareness}

In the present study, approximately $75 \%$ of the participants were already familiar with LARC. A study in the United States of 520 women aged 13-45 showed that a large number of 
respondents were familiar with LARC. They also knew a great deal about LARC, and three-quarters (76\%) had heard about LARC methods (Burns et al., 2015). However, another study carried out on female Hispanic adolescents aged 16 showed that only $50 \%$ of the participants had heard about LARC (Dobry et al., 2018).

In a cross-sectional convenience survey on Englishspeaking women who were referred to two sexual health clinics in Toronto, it was shown that $77 \%$ of the participants were already familiar with LARC (Buhling et al., 2014). Finally, a study conducted on $70 \%$ of a sample of Australian men and women aged 18-50 years old indicated that they had already been familiarized with LARC (17). All these studies are in agreement with the present study.

\section{LARC Usage}

Even though the participants were mostly aware of LARC, only $8.2 \%$ and $1.7 \%$ used IUDs and DMPA, respectively. According to a study carried out on 502 women aged 18-30 in the UK, in which they completed a cross-sectional online questionnaire, it was found that only $18 \%$ of the participants reported using LARC (Bracken \& Graham, 2014).

The number of married women participants using LARC methods was generally higher in France (27\%), Russia (32\%), Austria (23\%) and Germany (11\%) than United States (10\%) and Australia (7\%) (16).

A descriptive cross-sectional study in Turkey investigated the use of IUDs in a total sample of 106,669 women aged 15-49 years old. It was found that $25 \%$ of the participants used IUDs (Çalikoğlu et al., 2018).

\section{LARC Reliability}

Our study showed that $30 \%$ of the participants believed that the use of IUDs was reliable, while only $12 \%$ stated that DMPA was reliable. In a survey carried out in Australia, $46 \%$ of the participants believed that IUD was reliable (17).

\section{LARC as a Choice}

Various factors influenced the decision of one method of LARC over the other. These variables include religion, several marriages, partner's age, having a current contraception method, not having consulted with health professionals about contraception, a history of unwanted pregnancy, age of the participant, and the education level of participant and his/her spouse.

In a study carried out on 3155 participants by Jacqueline Coombes et al., it was found that the history of pregnancy was strongly associated with the increased use of LARC in Australian women (Coombe et al., 2017). A study on the relationship between LARC and parity has also suggested that women who have had one or more children were more likely to use these methods (Cea Soriano et al., 2014). The National Survey of Family Growth conducted a study (2011) on 7,643 women aged 15-44 years old. It was found that women who had given birth once or twice were more likely to use LARC (Hoopes et al., 2018; Kavanaugh et al., 2011). Our study suggests that nulliparous and teenagers did not use LARC methods. Instead, they usually used short-acting methods such as pills, condoms and natural methods.
The type of religion is an essential factor which could influence the results of this study. Since long-acting methods can change the bleeding patterns of menstruation (amenorrhea (Azmat et al., 2012), hypomenorrhea and menometrorrhagia (Kaneshiro \& Aeby, 2010), they may limit a woman's relationships and her ability to do domestic chores (Bateson et al., 2017). Intercourse during menstruation is considered forbidden in Islam (in both Shia and Sunni sects), and in some more conservative populations, it is reflected as a great sin (Soltani, 2005). In addition, some cultural and religious beliefs prevent menstruating women from participating in worship activities, including walking into a mosque or reading Quran (Salem \& Setty, 2006). When a woman on menstruation is socially and religiously considered as a sexual, dirty, shameful, dangerous, and scary creature that is also unbalanced in terms of mood, mad and inconsistent in decision-making, she sees herself highly vulnerable in experiencing menstruation. When blood flows inside the body, it is clean and the sign of life, but once it exits the body, it is considered unclean, and the body gets dirty. The release of menstrual discharge is thought to be insanitary, and a woman who is not on her menstruation is regarded as sanitary and a healthy creature (Zendehdel \& Elyasi, 2018). These reasons may lead to a reduction in the use of LARC. Our results showed that Shia women were more likely to make use of long-acting methods compared to Sunni women, which might be due to stricter religious rules in the Sunni sect.

Another critical finding which needs to be taken into consideration is the number of marriages. Women who were married more than once reported that long-acting methods were less favoured; this might be due to the desire to experience more stable families by having children. It has also been suggested that relationship status can influence the use of LARC. According to a study by Kavanaugh and Coombe, women who were in a cohabitating relationship or those who were engaged or married were more likely to use LARC than single women or women in non-cohabitating relationships (Kavanaugh et al., 2011; Salem \& Setty, 2006).

Women with younger husbands (24-40 years old) did not use long-lasting methods. Given the fact that the marriage age has increased among Iranian men and women over the past number of years, couples have less time for safe pregnancies. For this reason, they do not use long-lasting methods to complete their family as soon as possible. This result was not obtained in similar studies.

According to the researchers' findings, men and women who did not use contraception during their sexual relationships did not tend to use long-lasting methods. Infertility and emotional distance or disease are among the main reasons why the couples avoided sexual relations and consequently avoided contraception, including long-term methods.

The results of this study showed that those participants who had not consulted with health professionals did not have enough information about LARC methods and chose this method less than other contraception methods. The findings of the present study agree well with those of Daniele et al., Nansseu et al., Santoso and Surya (Coombe et al., 2016; Daniele et al., 2017; Zendehdel \& Elyasi, 2018). 
According to the results of this study, men and women who had experienced unwanted pregnancy were referred to health centers to receive advice on safe prevention and consequently became familiar with long-term preventive measures. This is how the couples avoided further pregnancies. It was mentioned in one other article that the fact that LARC is highly effective could be attractive for women, particularly for those who want to avoid unwanted pregnancy (30).

The present study shows that age plays an essential role in choosing LARC over other contraceptive methods: older men and women regarded IUDs and DMPA as reliable methods. The findings of the present study agree well with other reviews in the USA and France (Hoopes et al., 2018; Nansseu et al., 2015; Santoso et al., 2017).

The level of education is another criterion that needs to be taken into account. Men and women who had at least a diploma or degrees beyond the diploma used LARC methods less than those with lower levels of education. In addition, Kavanaugh et al. and Goldstone et al. also showed that a higher level of education is associated with decreased use of LARC (Daniels et al., 2014; Kavanaugh et al., 2011). The reason why women with higher levels of schooling avoid fertility using contraception methods other than LARC can be attributed to the fact that they are fully aware of the methods of prevention and do not require long-term methods.

\section{STRENGTHS AND WEAKNESSES OF THE STUDY}

The advantage of the present study was the relatively large number of women and men recruited. This was the first study of its kind in Iran. It was also the first study in Iran in which men were asked about long-term prevention methods.

The present study provided male participants with an opportunity to obtain thorough information on the LARC methods and the benefits of long-term effects of LARC.

The cross-sectional nature of this study could be considered a limitation. The participants in this study were both Iranians and Afghans. Owing to their lower socioeconomic status and lack of access to facilities, Afghans are classified as a particular group. The number of Afghan participants (85 women and 14 men) was insufficient for a separate analysis. Therefore, this study cannot be generalized to the Iranian population. Nevertheless, the fact is that Afghan immigrants have set down roots in Tehran and are key users of health care services.

\section{CONCLUSION}

The present study showed that men and women are familiar with LARC methods, but few believe that these methods are reliable. Owing to the changes in the Iranian family planning policy since 2014 and the elimination of teaching methods for preventing health education programs, modern methods of contraception are not uniformly accepted. The Iranian family planning program needs to be supported by the authorities to strengthen the pillars of our Islamic society.
The newly-wed Iranian men and women who are ready to start a family should be given access to information about the importance of family, the proper timing of pregnancy, and suitable spacing between children. Strategically-timed details on the right time to use LARC can be crucial for family development objectives.

\section{USE OF CLINICAL RESEARCH}

Training on methods of preventing pregnancy needs to be incorporated in the educational programs of health centers, as well as other scientific and cultural centers. Training for men must also be a part of the curriculum. In all dissemination of information by clinicians about preventive methods, obtaining feedback from the client is recommended.

\section{LIST OF ABBREVIATION}
LARC : long-acting reversible contraceptives
DMPA : Depo Medroxyprogesterone Acetate
TFR : Total Fertility Rate
IUD : Intrauterine Device
OR : Adjusted Odd Ratios
CI : Confidence Interval

\section{ACKNOWLEDGEMENTS}

The authors would like to thank Mazandaran University of Medical Sciences for the approval of ethical clearance and funding support of this study. We also thank Tehran University of Medical Sciences and selected health centers of the south of Tehran for facilitating this study. The authors also forward their gratitude to participants, data collectors, and supervisors who participated in the study.

\section{REFERENCES}

Azmat, S. K., Shaikh, B. T., Hameed, W., Bilgrami, M., Mustafa, G., et al. (2012). Rates of IUCD discontinuation and its associated factors among the clients of a social franchising network in Pakistan. BMC women's health, 12, 8. https://doi.org/10.1186/1472-6874-12-8

Bateson, D., Kang, S., Paterson, H., \& Singh, K. (2017). A review of intrauterine contraception in the Asia-Pacific region. Contraception, 95, 40-49. https://doi.org/10.1016/ j.contraception.2016.08.008

Bracken, J., \& Graham, C. A. (2014). Young women's attitudes towards, and experiences of, long-acting reversible contraceptives. The European Journal of Contraception \& Reproductive Health Care, 19, 276-284. https://doi.org/10.3109/13625187.2014.917623

Brown, S. S., \& Eisenberg, L. (1995). The best intentions: Unintended pregnancy and the well-being of children and families. National Academies Press. 
Buhling, K. J., Zite, N. B., Lotke, P., \& Black, K. (2014). Worldwide use of intrauterine contraception: a review. Contraception, 89, 162-173. https://doi.org/10.1016/ j.contraception.2013.11.011

Burns, B., Grindlay, K., \& Dennis, A. (2015). Women's awareness of, interest in, and experiences with long-acting reversible and permanent contraception. Women's Health Issues, 25, 224-231. https://doi.org/10.1016/j.whi.2014. 12.006

Çalikoğlu, E. O., Yerli, E. B., Kavuncuoğlu, D., Yılmaz, S., Koşan, Z., \& Aras, A. (2018). Use of Family Planning Methods and Influencing Factors Among Women in Erzurum. Medical science monitor: international medical journal of experimental and clinical research, 24, 5027. https://doi.org/10.12659/MSM.908388

Cea Soriano, L., Wallander, M.-A., Andersson, S., Filonenko, A., \& García Rodríguez, L. A. (2014). Use of long-acting reversible contraceptives in the UK from 2004 to 2010: an analysis using the health improvement network database. The European Journal of Contraception \& Reproductive Health Care, 19, 439-447. https://doi.org/10.3109/ 13625187.2014.948613

Committee A. (2009). Opinion no. 450: increasing use of contraceptive implants and intrauterine devices to reduce unintended pregnancy. Obstet Gynecol., 114, 1434-1438. https://doi.org/10.1097/AOG.0b013e3181c6f965

Coombe, J., Harris, M. L., \& Loxton, D. (2017). Who uses longacting reversible contraception? Profile of LARC users in the CUPID cohort. Sexual \& Reproductive Healthcare, 11, 19-24. https://doi.org/10.1016/j.srhc.2016.09.003

Coombe, J., Harris, M. L., Wigginton, B., Lucke, J. C., \& Loxton, D. (2016). Contraceptive use at the time of unintended pregnancy: Findings from the contraceptive use, pregnancy intention and decisions study. Australian family physician, 45, 842 .

Curtis, K. (2010). The World Health Organization's Medical eligibility criteria for contraceptive use: example of a national adaptation. IPPF Medical Bulletin, 44, 3-4.

Daniele, M. A., Cleland, J., Benova, L., \& Ali, M. (2017). Provider and lay perspectives on intra-uterine contraception: a global review. Reproductive health, 14, 119. https://doi.org/10.1186/s12978-017-0380-8

Daniels, K., Daugherty, J. D., \& Jones, J. (2014). Current contraceptive status among women aged 15-44: the United States, 2011-2013.

Dobry, A., Rojas-Mendez, P., Sanchez, S., \& Reddy, S. (2018). Influence of Acculturation in Knowledge and Acceptability of Intrauterine Devices in Hispanic Adolescents. Journal of Pediatric and Adolescent Gynecology, 31, 172. https://doi.org/10.1016/j.jpag.2018.02.037

Eeckhaut, M. C., Sweeney, M. M., \& Gipson, J. D. (2014). Who Is Using Long-Acting Reversible Contraceptive Methods? Findings from Nine Low-Fertility Countries. Perspectives on sexual and reproductive health, 46, 149-155. https://doi.org/10.1363/46e1914

Erfani, A. (2013). Fertility in Tehran city and Iran: rates, trends and differentials. Population Studies, 1, 87-107.
Goldstone, P. (2014). Factors predicting uptake of long-acting reversible methods of contraception among women presenting for abortion. America (North and South), 77, 1.2. https://doi.org/10.5694/mja14.00011

Group, L.-A. R. C. W. (2009). ACOG Committee Opinion no. 450: Increasing the use of contraceptive implants and intrauterine devices to reduce unintended pregnancy. Obstet Gynecol., 114, 1434-1438. https://doi.org/10.1097/ AOG.0b013e3181c6f965

Holton, S., Rowe, H., Kirkman, M., Jordan, L., McNamee, K., Bayly, C., et al. (2016). Long-acting reversible contraception: Findings from the Understanding Fertility Management in Contemporary Australia survey. The European Journal of Contraception \& Reproductive Health Care, 21, 116-131. https://doi.org/10.3109/13625187.2015. 1052394

Hoopes, A. J., Teal, S. B., Akers, A. Y., \& Sheeder, J. (2018). Low Acceptability of Certain Contraceptive Methods among Young Women. Journal of Pediatric and Adolescent Gynecology, 31, 274-280. https://doi.org/10.1016/j.jpag. 2017.11.008

Jalal Abbasi-Shavazi, M., \& McDonald, P. (2006). Fertility decline in the Islamic Republic of Iran: 1972-2000. Asian Population Studies, 2, 217-237. https://doi.org/10.1080/ 17441730601073789

Kaneshiro, B., \& Aeby, T. (2010). Long-term safety, efficacy, and patient acceptability of the intrauterine Copper T380A contraceptive device. International journal of women's health, 2, 211. https://doi.org/10.2147/IJWH.S6914

Kavanaugh, M. L., Jerman, J., Hubacher, D., Kost, K., \& Finer, L. B. (2011). Characteristics of women in the United States who use long-acting reversible contraceptive methods. Obstetrics \& Gynecology, 117, 1349-1357. https://doi.org/ 10.1097/AOG.0b013e31821c47c9

Likis, F. E. (2014). A new year's resolution: Promoting longacting reversible contraception. Journal of midwifery \& women's health, 59, 1-2. https://doi.org/10.1111/ jmwh.12181

Mehryar, A., Delavar, B., Farjadi, G., Hosseini-Chavoshi, M., Naghavi, M., \& Tabibian, M. (2001). Iranian miracle: how to raise contraceptive prevalence rate to above $70 \%$ and cut TFR by two-thirds in less than a decade. IUSSP Conference, p. 18-24.

Moosazadeh, M., Nekoei-moghadam, M., Emrani, Z., \& Amiresmaili, M. (2014). Prevalence of unwanted pregnancy in Iran: a systematic review and meta-analysis. The International journal of health planning and management, 29(3). https://doi.org/10.1002/hpm.2184

Moreau, C., Bohet, A., Hassoun, D., Teboul, M., Bajos, N., \& Group, F. W. (2013). Trends and determinants of use of long-acting reversible contraception use among young women in France: results from three national surveys conducted between 2000 and 2010. Fertility and sterility, 100, 451-458. https://doi.org/10.1016/j.fertnstert.2013.04. 002 
Motlaq, M. E., Eslami, M., Yazdanpanah, M., \& Nakhaee, N. (2013). Contraceptive use and unmet need for family planning in Iran. International Journal of Gynecology \& Obstetrics, 121(2), 157-161. https://doi.org/10.1016/j.ijgo. 2012.11.024

Namazi, A. (2015). The investigation of demographic variables associated with the phenomenon of unwanted pregnancy in Rasht. Nursing of the Vulnerables, 2, 29-38.

Nansseu, J. R. N., Nchinda, E. C., Katte, J.-C., Nchagnouot, F. M, \& Nguetsa, G. D. (2015). Assessing the knowledge, attitude and practice of family planning among women living in the Mbouda health district, Cameroon. Reproductive health, 12(1), 92. https://doi.org/10.1186/ s12978-015-0085-9

Peipert, J. F., Zhao, Q., Allsworth, J. E., Petrosky, E., Madden, T., Eisenberg, D., et al. (2011). Continuation and satisfaction of reversible contraception. Obstetrics and gynecology, 117, 1105. https://doi.org/10.1097/AOG. Ob013e31821188ad

Salem, R., \& Setty, V. (2006). When contraceptives change monthly bleeding: How family planning providers and programs can help clients choose and use suitable methods. Population Reports Series J: Family Planning Programs, 1-20.
Santoso, B. I., \& Surya, R. (2017). Knowledge, attitude, and practice of contraception among pregnant women in Ende districet, east Nusa Tennaggara, Indonesia. J South Asian Feder Obst Gynae., 9, 110-118. https://doi.org/10.5005/jpjournals-10006-1470

Sedgh, G., Singh, S., \& Hussain, R. (2014). Intended and unintended pregnancies worldwide in 2012 and recent trends. Studies in family planning, 45, 301-314. https://doi.org/10.1111/j.1728-4465.2014.00393.x

Simbar, M. (2010). Achievements of the family planning program in Iran. Journal of School of Public Health \& Institute of Public Health Research, 7(4).

Simbar, M. (2012). Achievements of the Iranian family planning programmes 1956-2006. Eastern Mediterranean health journal, 18(3). https://doi.org/10.26719/2012.18.3. 279

Soltani S. (2005). The sin of women in Ardavirafnameh.

Zaheri, F., Ranaie, F., Karimeh, R., \& Shahoi, R. (2015). Unwanted pregnancy and associated factors among pregnant women who referred to Sanandaj health centers in 2011. The Iranian Journal of Obstetrics, Gynecology and Infertility, 17, 10-15.

Zendehdel, M., \& Elyasi, F. (2018). Biopsychosocial etiology of premenstrual syndrome: A narrative review. Journal of family medicine and primary care, 7, 346. https://doi.org/10.4103/jfmpc.jfmpc_336_17 